\title{
Regular Resistor Lattice Networks in Two Dimensions (Archimedean Lattices)
}

\author{
M. Q. Owaidat ${ }^{1}$ \\ ${ }^{1}$ Al-Hussein Bin Talal University, Jordan \\ Correspondence: M. Q. Owaidat, Al-Hussein Bin Talal University, Jordan. E-mail: owaidat@ahu.edu.jo
}

Received: August 1, 2014 Accepted: August 23, 2014 Online Published: September 14, 2014

doi:10.5539/apr.v6n5p100 URL: http://dx.doi.org/10.5539/apr.v6n5p100

\begin{abstract}
In this paper, we investigate the two-vertex resistance on four Archimedean lattices. This technique is based on the Green's function corresponding to the Laplacian matrix of the lattice. The Laplacian matrix of the resistor network is determined by applying basic principles (Kirchhoff's and Ohm's laws) in electrical circuit analysis, and Fourier transforms of the electric potential and current. We present some numerical results regarding the resistance between nearby lattice vertices.
\end{abstract}

Keywords: regular resistor lattice; Green's function; two-vertex resistance; Archimedean lattices; Fourier transform

\section{Introduction}

Since the well-known work by (Kirchhoff, 1847) there have been several investigations on the problem of determining the effective resistance between any two vertices in infinite resistor networks (Venezian, 1994; Atkinson \& van Steenwijk, 1999; Jeng, 2004). One of the most approaches has been used to study this problem is the lattice Green's function. This method was recently introduced by (Cserti, 2000) for perfect lattices and developed by (Cserti, D'avid \& Pir'oth, 2002) for perturbed lattices. More recently, Cserti, Sz'echenyi, \& D'avid (2011) generalized the method in Cserti (2000) to any lattice structure that is a uniform tiling of space with resistors. Based on the lattice Green's method numerous applications were carried out in Refs. (Owaidat, Hijjawi \& Khalifeh, 2010(2), 2012, 2014; Owaidat, 2012, 2013; Owaidat, Hijjawi, Asad, \& Khalifeh, 2013; Asad, Diab, Owaidat, Hijjawi, \& Khalifeh, 2014).

The case of finite resistor networks was considered by (Wu, 2004) and extended to impedance networks in Ref. (Tzeng \& Wu, 2006). In this paper we apply the technique of Ref. (Cserti et al., 2011) to the following four Archimedean lattices, $\left(3^{3} .4^{2}\right),\left(3^{2} .4 .3 .4\right),\left(3^{4} .6\right)$ and (3.4.6.4), that have not been studied before, and obtain the resistance between any two vertices in the networks.

The paper is arranged as follows. In section 2 we briefly review the general formulation given in Ref. (Cserti et al., 2011)for obtaining the general formula of the effective resistance between any nodes in any lattice structure of uniform tilings of resistors. In section 3 we apply the formulation to compute the vertex to vertex resistance in the Archimedean lattices that mentioned above and present some numerical results regarding the resistance between certain pairs of vertices. In section 4 we give a short conclusion and discussion.

\section{Review the Formulation}

In this section, elements of the methodology of the two point resistance are briefly reviewed (for more details see Cserti et al., 2011).

Consider a resistor lattice network structure which is a periodic lattice of $d$-dimensional space with $N_{1}, N_{2}, \ldots, N_{d}$ unit cells along each unit cell vectors $\mathbf{a}_{1}, \mathbf{a}_{2}, \ldots, \mathbf{a}_{d}$. If the unit cell containing $s$ sites (vertices) numbered by $\alpha=1,2, \ldots, s$ then there are $s N_{1} N_{2} \ldots N_{d}$ vertices in the lattice. Let $\{\mathbf{r} ; \alpha\}$ denote any vertex, thus the unit cell and the lattice site can be specified $\mathrm{b} \mathbf{r}=\left(\ell_{1} \mathbf{a}_{1}, \ell_{2} \mathbf{a}_{2}, \ldots, \ell_{d} \mathbf{a}_{d}\right)$ and $\alpha$ respectively, where $\ell_{1}, \ell_{2}, \ldots, \ell_{d}$ are any integers. Assume, without lose of generality, that each resistor has of resistance $R$.

Denote the electric potential and current at vertex $\{\mathbf{r} ; \alpha\}$ by $V_{\alpha}(\mathbf{r})$ and $I_{\alpha}(\mathbf{r})$ respectively. Applying Kirchhoff's current and Ohm's laws at site $\{\mathbf{r} ; \alpha\}$, the currents $I_{\alpha}(\mathbf{r})$ in the unit cell can be written in the form: 


$$
\sum_{\mathbf{r}^{\prime}, \beta} L_{\alpha \beta}\left(\mathbf{r}, \mathbf{r}^{\prime}\right) V_{\beta}\left(\mathbf{r}^{\prime}\right)=-R I_{\alpha}(\mathbf{r})
$$

where $L_{\alpha \beta}\left(\mathbf{r}, \mathbf{r}^{\prime}\right)$ is the Laplacian matrix of the network. The potential and current at vertex $\{\mathbf{r} ; \alpha\}$ can be written in terms of their Fourier transforms:

$$
V_{\alpha}(\mathbf{r})=\Omega_{0} \int_{-\pi / a_{1}}^{\pi / a_{1}} \frac{d k_{1}}{2 \pi} \ldots \int_{-\pi / a_{d}}^{\pi / a_{d}} \frac{d k_{d}}{2 \pi} V_{\alpha}(\mathbf{k}) \mathrm{e}^{i \mathbf{k . r}},
$$

and

$$
I_{\alpha}(\mathbf{r})=\Omega_{0} \int_{-\pi / a_{1}}^{\pi / a_{1}} \frac{d k_{1}}{2 \pi} \ldots \int_{-\pi / a_{d}}^{\pi / a_{d}} \frac{d k_{d}}{2 \pi} I_{\alpha}(\mathbf{k}) \mathrm{e}^{i \mathbf{k} . \mathbf{r}}
$$

where $\Omega_{0}$ is volume of unit cell and $\mathbf{k}$ is the reciprocal lattice vector in first Brilliuon zone. Using equations (2) and (3), equation (1) may be written as

$$
\mathbf{L}(\mathbf{k}) \mathbf{V}(\mathbf{k})=-R \mathbf{I}(\mathbf{k})
$$

where $\mathbf{L}(\mathbf{k})$ is the Fourier transform of the Laplacian matrix ( $\mathrm{s}$ by s matrix) and $\mathbf{V}(\mathbf{r}), \mathbf{I}(\mathbf{k})$ are the Fourier transforms (column matrices) of the potential and current. The Fourier transform of the Green's function is given by

$$
\mathbf{L}(\mathbf{k}) \mathbf{G}(\mathbf{k})=-\mathbf{I}
$$

where $\mathbf{I}$ is s by s identity matrix.

From equations (4) and (5) we have

$$
\mathbf{V}(\mathbf{k})=R \mathbf{G}(\mathbf{k}) \mathbf{I}(\mathbf{k})
$$

Now the resistance between the vertices $\left\{\mathbf{r}_{1} ; \alpha\right\}$ and $\left\{\mathbf{r}_{2} ; \beta\right\}$, is given by

$$
R_{\alpha \beta}\left(\mathbf{r}_{1}, \mathbf{r}_{2}\right)=\frac{V_{\alpha}\left(\mathbf{r}_{1}\right)-V_{\beta}\left(\mathbf{r}_{2}\right)}{I}
$$

The computation of the two-point resistance is now reduced to solving equation (1) or (6) for $V_{\alpha}(\mathbf{r})$ with the current distribution at any vertex is given by

$$
I_{v}(\mathbf{r})=\left\{\begin{array}{l}
I, v=\alpha \text { and } \mathbf{r}=\mathbf{r}_{1} \\
-I, \nu=\beta \text { and } \mathbf{r}=\mathbf{r}_{2} \\
0, \text { otherwise }
\end{array}\right.
$$

Therefore, combining equations (2), ( 6) and (8) the electrical potential distribution at arbitrary vertex of the lattice is

$$
V_{\mu}(\mathbf{r})=\Omega_{0} R I \int_{-\pi / a_{1}}^{\pi / a_{1}} \frac{d k_{1}}{2 \pi} \ldots \int_{-\pi / a_{d}}^{\pi / a_{d}} \frac{d k_{d}}{2 \pi}\left(G_{\mu \alpha}(\mathbf{k}) \mathrm{e}^{\mathbf{i k} .\left(\mathbf{r}-\mathbf{r}_{1}\right)}-G_{\mu \beta}(\mathbf{k}) \mathrm{e}^{\mathbf{i k} .\left(\mathbf{r}-\mathbf{r}_{2}\right)}\right)
$$

Substituting potential distribution given above into equation (7), the two-node resistance can be written as

$$
R_{\alpha \beta}\left(\mathbf{r}_{1}, \mathbf{r}_{2}\right)=\Omega_{0} R \int_{-\pi / a_{1}}^{\pi / a_{1}} \frac{d k_{1}}{2 \pi} \ldots \int_{-\pi / a_{d}}^{\pi / a_{d}} \frac{d k_{d}}{2 \pi}\left(G_{\alpha \alpha}(\mathbf{k})+G_{\beta \beta}(\mathbf{k})-G_{\alpha \beta}(\mathbf{k}) \mathrm{e}^{-\mathbf{i k}\left(\mathbf{r}_{2}-\mathbf{r}_{1}\right)}-G_{\beta \alpha}(\mathbf{k}) \mathrm{e}^{\mathrm{ik}\left(\mathbf{r}_{2}-\mathbf{r}_{1}\right)}\right)
$$

It has been pointed out in (Cserti et al.,2011) that the lattice structure can be deformed into $d$ - dimensional hypercubic lattice without changing the two point resistance of a resistor network. For hypercubic lattice the unit cell vectors are orthogonal and have the same magnitude. Thus, if one makes the transformations $\mathbf{r}_{2}-\mathbf{r}_{1}=\left(\ell_{1} \mathbf{a}_{1}, \ell_{2} \mathbf{a}_{2}, \ldots, \ell_{d} \mathbf{a}_{d}\right)$ and $\mathbf{k} . \mathbf{a}_{\mathbf{i}}=\theta_{i}$ with $\Omega_{0}=a^{d}$ then, equation (10) becomes

$$
R_{\alpha \beta}\left(\ell_{1}, . ., \ell_{d}\right)=R \int_{-\pi}^{\pi} \frac{d \theta_{1}}{2 \pi} . . \int_{-\pi}^{\pi} \frac{d \theta_{d}}{2 \pi}\left\{\begin{array}{c}
\left.G_{\alpha \alpha}\left(\theta_{1}, . ., \theta_{d}\right)-G_{\alpha \beta}\left(\theta_{1}, . ., \theta_{d}\right) \mathrm{e}^{-\mathrm{i}\left(\ell_{1} \theta_{1}+\ell_{2} \theta_{2}+. . \theta_{d} \ell\right.}\right) \\
+G_{\beta \beta}\left(\theta_{1}, . ., \theta_{d}\right)-G_{\beta \alpha}\left(\theta_{1}, . ., \theta_{d}\right) \mathrm{e}^{\mathrm{i}\left(\ell_{1} \theta_{1}+\ell_{2} \theta_{2}+. . \theta_{d} \ell_{d}\right)}
\end{array}\right\}
$$


For two dimensional lattices the resistance between the origin $\{\mathbf{0} ; \alpha\}$ and vertex $\left\{\ell_{1}, \ell_{2} ; \beta\right\}$ is

$$
R_{\alpha \beta}\left(\ell_{1}, \ell_{2}\right)=\frac{R}{(2 \pi)^{2}} \int_{-\pi-\pi}^{\pi} \int_{-\pi}^{\pi}\left\{\begin{array}{l}
G_{\alpha \alpha}\left(\theta_{1}, \theta_{2}\right)-G_{\alpha \beta}\left(\theta_{1}, \theta_{2}\right) \mathrm{e}^{-\mathrm{i}\left(\ell_{1} \theta_{1}+\ell_{2} \theta_{2}\right)} \\
+G_{\beta \beta}\left(\theta_{1}, \theta_{2}\right)-G_{\beta \alpha}\left(\theta_{1}, \theta_{2}\right) \mathrm{e}^{\mathrm{i}\left(\ell_{1} \theta_{1}+\ell_{2} \theta_{2}\right)}
\end{array}\right\} d \theta_{1} d \theta_{2}
$$

\section{Two Dimensional Uniform Tilings (Archimedean Lattices)}

Archimedean lattices are well-known in the literature. There are eleven Archimedean lattices which are uniform tiling in two dimensions by regular polygons. The square, triangular and honeycomb lattices have been studied in Refs. (Atkinson \& Van Steenwijk, 1999; Cserti, 2000), the Kagomé and (4.8.8) lattices in (Cserti et al., 2011). In this section, we calculate the effective resistance between two arbitrary vertices in the four Archimedean networks; $\left(3^{3} \cdot 4^{2}\right),\left(3^{2} \cdot 4.3 .4\right),\left(3^{4} \cdot 6\right)$ and $(3.4 .6 .4)$.

$3.1\left(3^{3} \cdot 4^{2}\right)$ Lattice Network

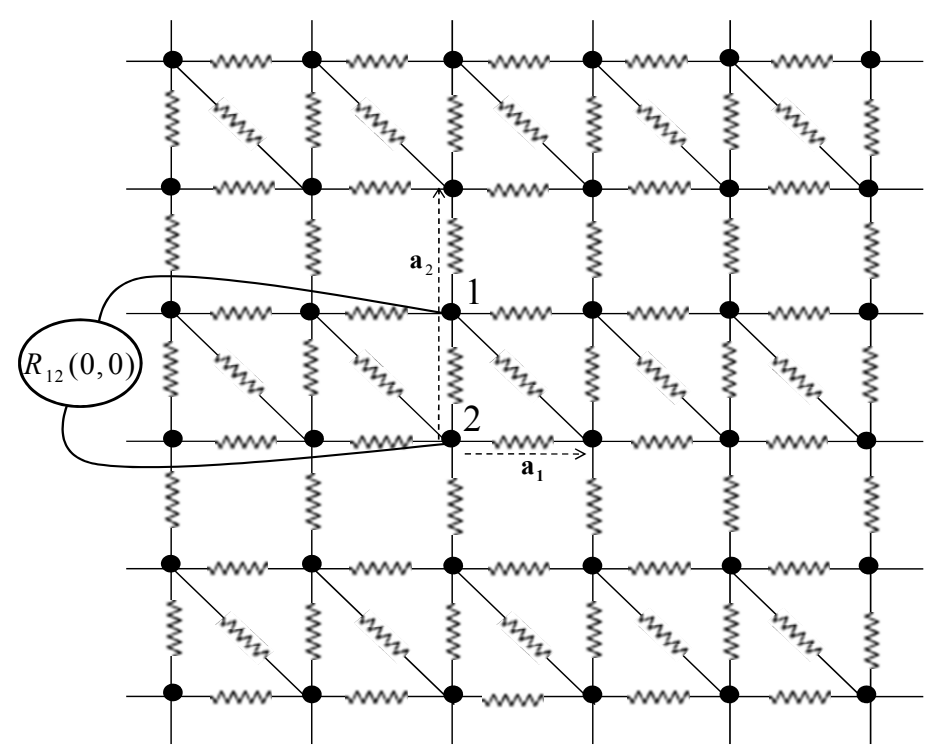

Figure 1.The resistor network of $\left(3^{3} \cdot 4^{2}\right)$ lattice

The $\left(3^{3} .4^{2}\right)$ network is shown in Figure 1, where the simplest unit cell contains two lattice sites labeled by $\alpha=1,2$ and the unit cell vectors are $\mathbf{a}_{1}$ and $\mathbf{a}_{2}$ as shown. According to Kirchhoff's and Ohm's laws, one can write the currents at the vertices $\{\mathbf{r} ; 1\}$ and $\{\mathbf{r} ; 2\}$ as following:

$$
\begin{gathered}
I_{1}(\mathbf{r})=\frac{V_{1}(\mathbf{r})-V_{1}\left(\mathbf{r}+\mathbf{a}_{1}\right)}{R}+\frac{V_{1}(\mathbf{r})-V_{1}\left(\mathbf{r}-\mathbf{a}_{1}\right)}{R}+\frac{V_{1}(\mathbf{r})-V_{2}(\mathbf{r})}{R}+\frac{V_{1}(\mathbf{r})-V_{2}\left(\mathbf{r}+\mathbf{a}_{1}\right)}{R}+\frac{V_{1}(\mathbf{r})-V_{2}\left(\mathbf{r}+\mathbf{a}_{2}\right)}{R}(13) \\
I_{2}(\mathbf{r})=\frac{V_{2}(\mathbf{r})-V_{1}(\mathbf{r})}{R}+\frac{V_{2}(\mathbf{r})-V_{1}\left(\mathbf{r}-\mathbf{a}_{1}\right)}{R}+\frac{V_{2}(\mathbf{r})-V_{1}\left(\mathbf{r}-\mathbf{a}_{2}\right)}{R}+\frac{V_{2}(\mathbf{r})-V_{2}\left(\mathbf{r}+\mathbf{a}_{1}\right)}{R}+\frac{V_{2}(\mathbf{r})-V_{2}\left(\mathbf{r}-\mathbf{a}_{1}\right)}{R}(14
\end{gathered}
$$

Substituting equations (2) and (3) into (13) and (14), the Laplacian matrix of the $\left(3^{3} \cdot 4^{2}\right)$ lattice, after changing $\mathbf{k} . \mathbf{a}_{i}=\theta_{i}(i=1,2)$, can be written as

$$
\mathbf{L}\left(\theta_{1}, \theta_{2}\right)=\left(\begin{array}{cc}
-5+2 \cos \theta_{1} & 1+e^{i \theta_{1}}+e^{i \theta_{2}} \\
1+e^{-i \theta_{1}}+e^{-i \theta_{2}} & -5+2 \cos \theta_{1}
\end{array}\right)
$$

and the lattice Green's function can be obtained from equation (5): 


$$
\mathbf{G}\left(\theta_{1}, \theta_{2}\right)=\frac{1}{\operatorname{det} \mathbf{L}\left(\theta_{1}, \theta_{2}\right)}\left(\begin{array}{cc}
-5+2 \cos \theta_{1} & 1+e^{i \theta_{1}}+e^{i \theta_{2}} \\
1+e^{-i \theta_{1}}+e^{-i \theta_{2}} & -5+2 \cos \theta_{1}
\end{array}\right)
$$

where

$$
\operatorname{det} \mathbf{L}\left(\theta_{1}, \theta_{2}\right)=24-22 \cos \theta_{1}-2 \cos \left(\theta_{1}-\theta_{2}\right)-2 \cos \theta_{2}+2 \cos 2 \theta_{1}
$$

is the determinant of the Laplacian matrix. Now the equivalent resistance between any two sites can be calculated from equation (12). As an example, the resistance between the origin $\{\mathbf{0} ; \alpha=1\}$ and the site $\{\mathbf{0} ; \beta=2\}$ (see figure 1) is given by

$$
R_{12}(0,0)=\frac{R}{4 \pi^{2}} \int_{-\pi}^{\pi} \int_{-\pi}^{\pi} \frac{\left(4-3 \cos \theta_{1}-\cos \theta_{2}\right) d \theta_{1} d \theta_{2}}{12-11 \cos \theta_{1}-\cos \theta_{2}-\cos \left(\theta_{1}-\theta_{2}\right)+\cos 2 \theta_{1}}
$$

The numerical calculation of this integral is $\mathrm{R}_{12}(0,0)=0.400648 \mathrm{R}$. Numerical values for some other resistances are displaced in Table 1.

Table 1. Numerical values of $R_{\alpha \beta}\left(\ell_{1}, \ell_{2}\right)$ in units of $R$ for a $\left(3^{3} \cdot 4^{2}\right)$ lattice

\begin{tabular}{cccc}
\hline$R_{\alpha \beta}\left(\ell_{1}, \ell_{2}\right)$ & $\begin{array}{c}\text { The value of } \\
R_{\alpha \beta}\left(\ell_{1}, \ell_{2}\right) \\
\text { in terms of } R\end{array}$ & $R_{\alpha \beta}\left(\ell_{1}, \ell_{2}\right)$ & $\begin{array}{c}\text { The value of } \\
R_{\alpha \beta}\left(\ell_{1}, \ell_{2}\right) \\
\text { in terms of } R\end{array}$ \\
\hline$R_{11}(0,0)$ & 0 & $R_{12}(0,0)$ & 0.400648 \\
$R_{11}(1,0)$ & 0.401606 & $R_{12}(1,0)$ & 0.340298 \\
$R_{11}(2,0)$ & 0.572593 & $R_{12}(2,0)$ & 0.508612 \\
$R_{11}(0,1)$ & 0.577461 & $R_{12}(0,1)$ & 0.417312 \\
$R_{11}(0,2)$ & 0.749104 & $R_{12}(0,2)$ & 0.742836 \\
$R_{11}(1,1)$ & 0.630838 & $R_{12}(1,1)$ & 0.635586 \\
$R_{11}(2,1)$ & 0.692814 & $R_{21}(-1,0)$ & 0.340298 \\
$R_{11}(1,-1)$ & 0.577461 & $R_{21}(1,0)$ & 0.623151 \\
$R_{11}(3,0)$ & 0.674959 & $R_{21}(0,1)$ & 0.778646 \\
$R_{11}(0,-1)$ & 0.577461 & $R_{21}(0,-1)$ & 0.417312 \\
\hline
\end{tabular}

$3.2\left(3^{2} .4 .3 .4\right)$ network

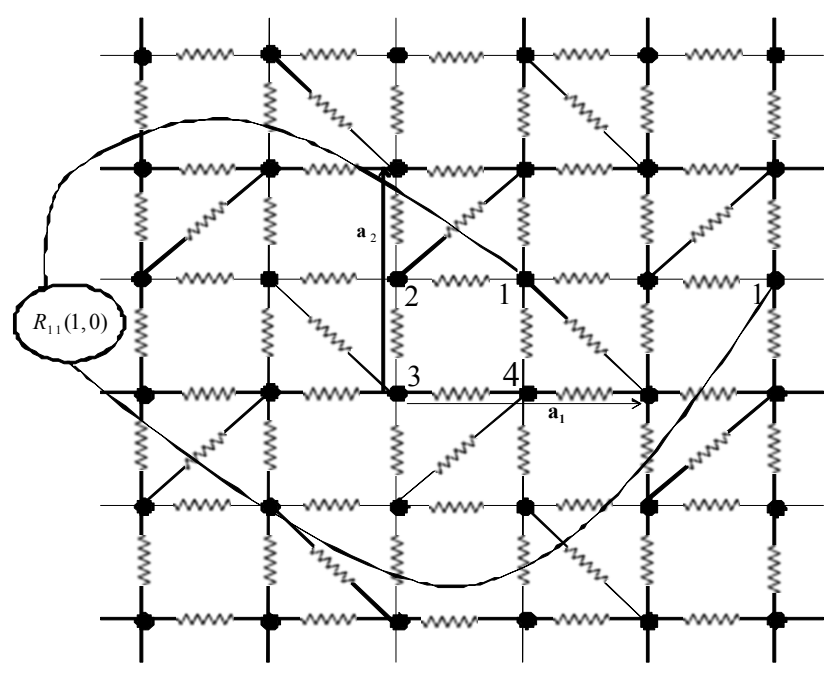

Figure 2.The resistor network of $\left(3^{2}\right.$. 4. 3. 4)lattice 
Figure 2 shows the $\left(3^{2} .4 .3 .4\right)$ network. The unit cell can be taken as a square containing four verticies labeled by $\alpha=1,2,3,4$ and the unit cell vectors are $\mathbf{a}_{1}$ and $\mathbf{a}_{2}$ as in Figure 2. Using Kirchhoff's and Ohm's laws the currents at sites $\{\mathbf{r} ; \alpha=1,2,3,4\}$ can be written as

$$
\begin{aligned}
& I_{1}(\mathbf{r})=\frac{V_{1}(\mathbf{r})-V_{2}(\mathbf{r})}{R}+\frac{V_{1}(\mathbf{r})-V_{2}\left(\mathbf{r}+\mathbf{a}_{1}\right)}{R}+\frac{V_{1}(\mathbf{r})-V_{3}\left(\mathbf{r}+\mathbf{a}_{1}\right)}{R}+\frac{V_{1}(\mathbf{r})-V_{4}(\mathbf{r})}{R}+\frac{V_{1}(\mathbf{r})-V_{4}\left(\mathbf{r}+\mathbf{a}_{2}\right)}{R} \\
& I_{2}(\mathbf{r})=\frac{V_{2}(\mathbf{r})-V_{1}(\mathbf{r})}{R}+\frac{V_{2}(\mathbf{r})-V_{1}\left(\mathbf{r}-\mathbf{a}_{1}\right)}{R}+\frac{V_{2}(\mathbf{r})-V_{3}(\mathbf{r})}{R}+\frac{V_{2}(\mathbf{r})-V_{3}\left(\mathbf{r}+\mathbf{a}_{2}\right)}{R}+\frac{V_{2}(\mathbf{r})-V_{4}\left(\mathbf{r}+\mathbf{a}_{2}\right)}{R} \\
& I_{3}(\mathbf{r})=\frac{V_{3}(\mathbf{r})-V_{1}\left(\mathbf{r}-\mathbf{a}_{1}\right)}{R}+\frac{V_{3}(\mathbf{r})-V_{2}(\mathbf{r})}{R}+\frac{V_{3}(\mathbf{r})-V_{2}\left(\mathbf{r}-\mathbf{a}_{2}\right)}{R}+\frac{V_{3}(\mathbf{r})-V_{4}(\mathbf{r})}{R}+\frac{V_{3}(\mathbf{r})-V_{4}\left(\mathbf{r}-\mathbf{a}_{1}\right)}{R} \\
& I_{4}(\mathbf{r})=\frac{V_{4}(\mathbf{r})-V_{1}(\mathbf{r})}{R}+\frac{V_{4}(\mathbf{r})-V_{1}\left(\mathbf{r}-\mathbf{a}_{2}\right)}{R}+\frac{V_{4}(\mathbf{r})-V_{2}\left(\mathbf{r}-\mathbf{a}_{2}\right)}{R}+\frac{V_{4}(\mathbf{r})-V_{3}(\mathbf{r})}{R}+\frac{V_{4}(\mathbf{r})-V_{3}\left(\mathbf{r}+\mathbf{a}_{1}\right)}{R}
\end{aligned}
$$

As in previous subsection the Fourier transform of the Laplacian matrix reads

$$
\mathbf{L}\left(\theta_{1}, \theta_{2}\right)=\left(\begin{array}{cccc}
-5 & 1+e^{i \theta_{1}} & e^{i \theta_{1}} & 1+e^{i \theta_{2}} \\
1+e^{-i \theta_{1}} & -5 & 1+e^{i \theta_{2}} & e^{i \theta_{2}} \\
e^{-i \theta_{1}} & 1+e^{-i \theta_{2}} & -5 & 1+e^{-i \theta_{1}} \\
1+e^{-i \theta_{2}} & e^{-i \theta_{2}} & 1+e^{i \theta_{1}} & -5
\end{array}\right)
$$

and its Green's function can be obtained from equation(5). Using equation (12) one can numerically calculate the resistance between any two sites. As an example, the resistance between nodes $\{\mathbf{0} ; \alpha=1\}$ and $\{\mathbf{0} ; \beta=2\}$ is given by

$$
R_{12}(0,0)=\frac{R}{4 \pi^{2}} \int_{-\pi}^{\pi} \int_{-\pi}^{\pi} \frac{\left(69-40 \cos \theta_{1}-20 \cos \theta_{2}-10 \cos \theta_{1} \cos \theta_{2}+\cos 2 \theta_{1}\right) d \theta_{1} d \theta_{2}}{170-72 \cos \theta_{1}-72 \cos \theta_{2}-28 \cos \theta_{1} \cos \theta_{2}+\cos 2 \theta_{1}+\cos 2 \theta_{2}}
$$

The numerical value of this integral is $R_{12}(0,0)=0.403775 R$. In Table 2 , we list numerical values for some extra resistances.

Table 2. Numerical values of $R_{\alpha \beta}\left(\ell_{1}, \ell_{2}\right)$ in units of $R$ for a $\left(3^{2} .4 .3 .4\right)$ lattice

\begin{tabular}{cccc}
\hline$R_{\alpha \beta}\left(\ell_{1}, \ell_{2}\right)$ & $\begin{array}{c}\text { Thevalueof } \\
R_{\alpha \beta}\left(\ell_{1}, \ell_{2}\right) \\
\text { intermsof } R\end{array}$ & $R_{\alpha \beta}\left(\ell_{1}, \ell_{2}\right)$ & $\begin{array}{c}\text { Thevalueof } \\
R_{\alpha \beta}\left(\ell_{1}, \ell_{2}\right) \\
\text { intermsof } R\end{array}$ \\
\hline$R_{11}(0,0)$ & 0 & $R_{12}(0,0)$ & 0.403775 \\
$R_{11}(1,0)$ & 0.564739 & $R_{13}(0,0)$ & 0.510759 \\
$R_{11}(2,0)$ & 0.730728 & $R_{34}(0,0)$ & 0.403775 \\
$R_{11}(0,1)$ & 0.564739 & $R_{23}(0,0)$ & 0.403775 \\
$R_{11}(0,2)$ & 0.730728 & $R_{24}(0,0)$ & 0.510759 \\
$R_{11}(1,1)$ & 0.651261 & $R_{14}(0,0)$ & 0.403775 \\
\hline
\end{tabular}

\section{$3.3\left(3^{4} .6\right)$ network}

Figure 3 shows the $\left(3^{4} .6\right)$ network. The unit cell is a hexagon containing six vertices labeled by $\alpha=1,2, \ldots, 6$. Using Kirchhoff's and Ohm's laws the currents at verices $\{\mathbf{r} ; \alpha=1,2, \ldots, 6\}$ can be written as

$$
\begin{aligned}
I_{1}(\mathbf{r})= & \frac{V_{1}(\mathbf{r})-V_{2}(\mathbf{r})}{R}+\frac{V_{1}(\mathbf{r})-V_{3}\left(\mathbf{r}+\mathbf{a}_{1}\right)}{R}+\frac{V_{1}(\mathbf{r})-V_{4}\left(\mathbf{r}+\mathbf{a}_{1}+\mathbf{a}_{2}\right)}{R} \\
& +\frac{V_{1}(\mathbf{r})-V_{5}\left(\mathbf{r}+\mathbf{a}_{1}+\mathbf{a}_{2}\right)}{R}+\frac{V_{1}(\mathbf{r})-V_{6}(\mathbf{r})}{R}
\end{aligned}
$$




$$
\begin{gathered}
I_{2}(\mathbf{r})=\frac{V_{2}(\mathbf{r})-V_{1}(\mathbf{r})+\frac{V_{2}(\mathbf{r})-V_{3}(\mathbf{r})}{R}+\frac{V_{2}(\mathbf{r})-V_{4}\left(\mathbf{r}+\mathbf{a}_{1}+\mathbf{a}_{2}\right)}{R}}{R} \\
+\frac{V_{2}(\mathbf{r})-V_{5}\left(\mathbf{r}+\mathbf{a}_{2}\right)}{R}+\frac{V_{2}(\mathbf{r})-V_{6}\left(\mathbf{r}+\mathbf{a}_{2}\right)}{R} \\
I_{3}(\mathbf{r})=\frac{V_{3}(\mathbf{r})-V_{1}\left(\mathbf{r}-\mathbf{a}_{1}\right)}{R}+\frac{V_{3}(\mathbf{r})-V_{2}(\mathbf{r})}{R}+\frac{V_{3}(\mathbf{r})-V_{4}(\mathbf{r})}{R} \\
I_{4}(\mathbf{r})=\frac{V_{4}(\mathbf{r})-V_{1}\left(\mathbf{r}-\mathbf{a}_{1}-\mathbf{a}_{2}\right)}{R}+\frac{V_{4}(\mathbf{r})-V_{2}\left(\mathbf{r}-\mathbf{a}_{1}-\mathbf{a}_{2}\right)}{R}+\frac{V_{4}(\mathbf{r})-V_{3}(\mathbf{r})}{R} \\
+\frac{V_{4}(\mathbf{r})-V_{5}(\mathbf{r})}{R}+\frac{V_{4}(\mathbf{r})-V_{6}\left(\mathbf{r}-\mathbf{a}_{1}\right)}{R}+\frac{V_{3}(\mathbf{r})-V_{6}\left(\mathbf{r}-\mathbf{a}_{1}\right)}{R} \\
I_{5}(\mathbf{r})= \\
\frac{V_{5}(\mathbf{r})-V_{1}\left(\mathbf{r}-\mathbf{a}_{1}-\mathbf{a}_{2}\right)}{R}+\frac{V_{5}(\mathbf{r})-V_{2}\left(\mathbf{r}-\mathbf{a}_{2}\right)}{R}+\frac{V_{5}(\mathbf{r})-V_{3}\left(\mathbf{r}-\mathbf{a}_{2}\right)}{R} \\
+\frac{V_{5}(\mathbf{r})-V_{4}(\mathbf{r})}{R}+\frac{V_{5}(\mathbf{r})-V_{6}(\mathbf{r})}{R} \\
I_{6}(\mathbf{r})=\frac{V_{6}(\mathbf{r})-V_{1}(\mathbf{r})}{R}+\frac{V_{6}(\mathbf{r})-V_{2}\left(\mathbf{r}-\mathbf{a}_{2}\right)}{R}+\frac{V_{6}(\mathbf{r})-V_{3}\left(\mathbf{r}+\mathbf{a}_{1}\right)}{R} \\
\quad+\frac{V_{6}(\mathbf{r})-V_{4}\left(\mathbf{r}+\mathbf{a}_{1}\right)}{R}+\frac{V_{6}(\mathbf{r})-V_{5}(\mathbf{r})}{R}
\end{gathered}
$$

Again the Fourier transform of the Laplacian matrix can be obtained as previously to be

$$
\mathbf{L}\left(\theta_{1}, \theta_{2}\right)=\left(\begin{array}{cccccc}
-5 & 1 & e^{i \theta_{1}} & e^{i\left(\theta_{1}+\theta_{2}\right)} & e^{i\left(\theta_{1}+\theta_{2}\right)} & 1 \\
1 & -5 & 1 & e^{i\left(\theta_{1}+\theta_{2}\right)} & e^{i \theta_{2}} & e^{i \theta_{2}} \\
e^{-i \theta_{1}} & 1 & -5 & 1 & e^{i \theta_{2}} & e^{-i \theta_{1}} \\
e^{-i\left(\theta_{1}+\theta_{2}\right)} & e^{-i\left(\theta_{1}+\theta_{2}\right)} & 1 & -5 & 1 & e^{-i \theta_{1}} \\
e^{-i\left(\theta_{1}+\theta_{2}\right)} & e^{-i \theta_{2}} & e^{-i \theta_{2}} & 1 & -5 & 1 \\
1 & e^{-i \theta_{2}} & e^{i \theta_{1}} & e^{i \theta_{1}} & 1 & -5
\end{array}\right)
$$

After obtaining the Green's function from equation (5), the resistance between any two sites can be calculated numerically from equation (12). As an example, the resistance between sites $\{\mathbf{0} ; \alpha=1\}$ and $\{1,0 ; \beta=1\}$ is given by

$$
\begin{aligned}
R_{11}(1,0) & =\frac{R}{4 \pi^{2}} \int_{-\pi}^{\pi} \int_{-\pi}^{\pi} \frac{\left(1724-140 \cos \theta_{1}-74 \cos \theta_{2}-212 \cos \left(\theta_{1}+\theta_{2}\right)-2 \cos \left(2 \theta_{1}+\theta_{2}\right)\left(1-\cos \theta_{1}\right) d \theta_{1} d \theta_{2}\right.}{\left\{\begin{array}{l}
2517-826 \cos \theta_{1}-826 \cos \theta_{2}-812 \cos \left(\theta_{1}+\theta_{2}\right)-14 \cos \left(2 \theta_{1}+\theta_{2}\right) \\
-14 \cos \left(\theta_{1}+2 \theta_{2}\right)-28 \cos \theta_{1} \cos \theta_{2}+\cos 2 \theta_{2}+\cos \left(2 \theta_{1}+2 \theta_{2}\right)
\end{array}\right\}} \\
& =0.583973 R
\end{aligned}
$$

In Table 3, we listed some resistance values near the origin. 


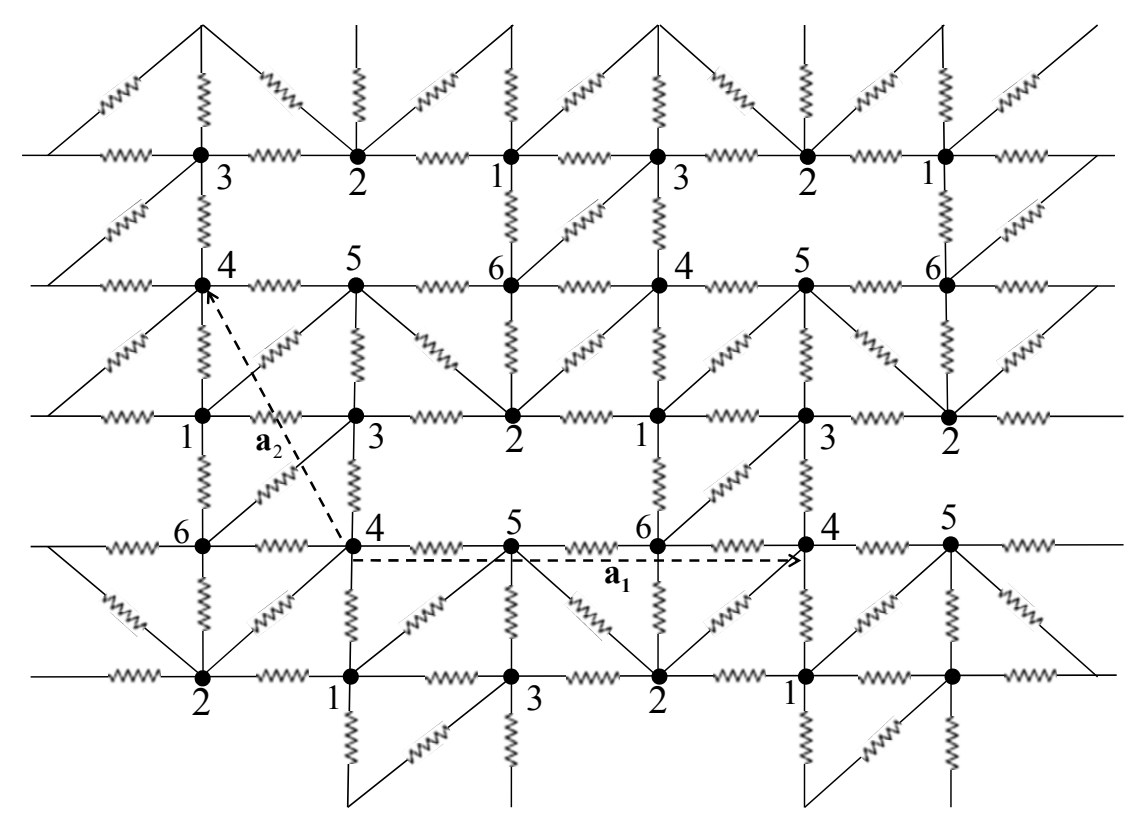

Figure 3.The resistor network of $\left(3^{4} .6\right)$ lattice

Table 3. Numerical values of $R_{\alpha \beta}\left(\ell_{1}, \ell_{2}\right)$ in units of $R$ for a (3 $3^{4}$.6) lattice

\begin{tabular}{cccc}
\hline$R_{\alpha \beta}\left(\ell_{1}, \ell_{2}\right)$ & $\begin{array}{c}\text { Thevalueof } \\
R_{\alpha \beta}\left(\ell_{1}, \ell_{2}\right) \\
\text { intermsof } R\end{array}$ & $R_{\alpha \beta}\left(\ell_{1}, \ell_{2}\right)$ & $\begin{array}{c}\text { Thevalueof } \\
R_{\alpha \beta}\left(\ell_{1}, \ell_{2}\right) \\
\text { intermsof } R\end{array}$ \\
\hline$R_{12}(0,0)$ & 0.365993 & $R_{26}(0,0)$ & 0.520674 \\
$R_{13}(0,0)$ & 0.511082 & $R_{34}(0,0)$ & 0.372814 \\
$R_{14}(0,0)$ & 0.601145 & $R_{35}(0,0)$ & 0.520674 \\
$R_{15}(0,0)$ & 0.553051 & $R_{36}(0,0)$ & 0.561246 \\
$R_{16}(0,0)$ & 0.548426 & $R_{45}(0,0)$ & 0.365993 \\
$R_{23}(0,0)$ & 0.376727 & $R_{46}(0,0)$ & 0.531172 \\
$R_{24}(0,0)$ & 0.55308 & $R_{56}(0,0)$ & 0.376727 \\
$R_{25}(0,0)$ & 0.583099 & $R_{11}(1,0)$ & 0.583973 \\
\hline
\end{tabular}

\section{4 (3.4.6.4) Network}

The (3.4.6.4) network is shown in Figure 4. Also the unit cell can be taken as a hexagon containing six lattice sites numbered by $\alpha=1,2, \ldots, 6$.

As in the previous subsections the Fourier transform of the Laplacian matrix of the (3.4.6.4) network can easily be obtained :

$$
\mathbf{L}\left(\theta_{1}, \theta_{2}\right)=\left(\begin{array}{cccccc}
-4 & 1 & e^{i \theta_{1}} & 0 & e^{i\left(\theta_{1}+\theta_{2}\right)} & 1 \\
1 & -4 & 1 & e^{i\left(\theta_{1}+\theta_{2}\right)} & 0 & e^{i \theta_{2}} \\
e^{-i \theta_{1}} & 1 & -4 & 1 & e^{i \theta_{2}} & 0 \\
0 & e^{-i\left(\theta_{1}+\theta_{2}\right)} & 1 & -4 & 1 & e^{-i \theta_{1}} \\
e^{-i\left(\theta_{1}+\theta_{2}\right)} & 0 & e^{-i \theta_{2}} & 1 & -4 & 1 \\
1 & e^{-i \theta_{2}} & 0 & e^{i \theta_{1}} & 1 & -4
\end{array}\right)
$$


The resistance between two arbitrary vertices can be computed from equation (12) . For example, the resistance between the origin $\{0 ; \alpha=1\}$ and the vertex $\{1,0 ; \beta=1\}$ is given by

$$
R_{11}(1,0)=\frac{R}{4 \pi^{2}} \int_{-\pi}^{\pi} \int_{-\pi}^{\pi} \frac{2\left(512-48 \cos \theta_{1}-32 \cos \theta_{2}-48 \cos \theta_{1} \cos \theta_{2}\right)\left(1-\cos \theta_{1}\right) d \theta_{1} d \theta_{2}}{\left\{\begin{array}{l}
1146-380 \cos \theta_{1}-380 \cos \theta_{2}-380 \cos \left(\theta_{1}+\theta_{2}\right)-4 \cos \left(2 \theta_{1}+\theta_{2}\right) \\
-4 \cos \left(\theta_{1}+2 \theta_{2}\right)-4 \cos \left(\theta_{1}-\theta_{2}\right)+2 \cos 2 \theta_{1}+2 \cos 2 \theta_{2}+2 \cos \left(2 \theta_{1}+2 \theta_{2}\right)
\end{array}\right\}}
$$

The numerical value of this resistance is $R_{11}(1,0)=0.763555 R$. However, some resistance values are listed in Table 4 .

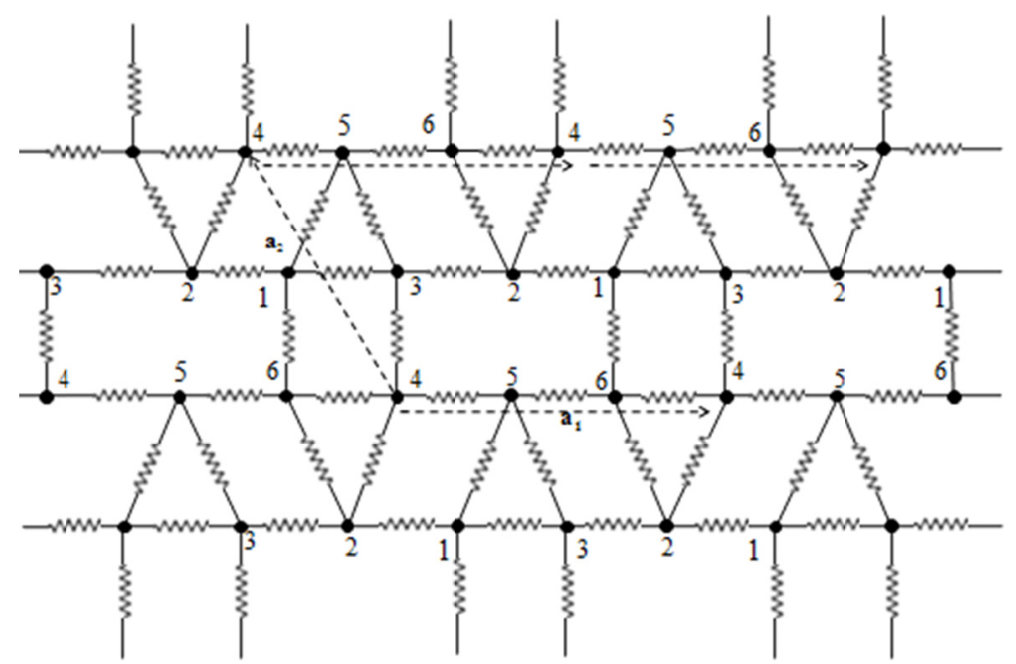

Figure 4.The resistor network of (3. 4. 6. 4) lattice

Table 4. Numerical values of $R_{\alpha \beta}\left(\ell_{1}, \ell_{2}\right)$ in units of $R$ for a (3. 4. 6. 4) lattice

\begin{tabular}{cccc}
\hline$R_{\alpha \beta}\left(\ell_{1}, \ell_{2}\right)$ & $\begin{array}{c}\text { Thevalueof } \\
R_{\alpha \beta}\left(\ell_{1}, \ell_{2}\right) \\
\text { intermsof } R\end{array}$ & $R_{\alpha \beta}\left(\ell_{1}, \ell_{2}\right)$ & $\begin{array}{c}\text { Thevalueof } \\
R_{\alpha \beta}\left(\ell_{1}, \ell_{2}\right) \\
\text { intermsof } R\end{array}$ \\
\hline$R_{12}(0,0)$ & 0.461578 & $R_{26}(0,0)$ & 0.474428 \\
$R_{13}(0,0)$ & 0.671497 & $R_{34}(0,0)$ & 0.474428 \\
$R_{14}(0,0)$ & 0.755492 & $R_{35}(0,0)$ & 0.671497 \\
$R_{15}(0,0)$ & 0.710417 & $R_{36}(0,0)$ & 0.715988 \\
$R_{16}(0,0)$ & 0.474428 & $R_{45}(0,0)$ & 0.461578 \\
$R_{23}(0,0)$ & 0.474428 & $R_{46}(0,0)$ & 0.671497 \\
$R_{24}(0,0)$ & 0.710417 & $R_{56}(0,0)$ & 0.4744278 \\
$R_{25}(0,0)$ & 0.755492 & $R_{11}(1,0)$ & 0.763555 \\
\hline
\end{tabular}

\section{Conclusion and Discussion}

We have determined the two-vertex resistance on periodic tilings in two dimensions (Archimedean lattices: $\left(3^{3} .4^{2}\right)$, $\left(3^{2} .4 .3 .4\right),\left(3^{4} .6\right)$ and $(3.4 .6 .4)$ with resistors. This problem has been made by using the lattice Green's function approach (Cserti et al., 2011). Some numerical results for resistance values near the networks origin are presented.

We believe that the Green's function method is a highly effective approach for the present problem and for any periodic tilings of resistors, even in cases when other methods (Venezian, 1994; Atkinson \& van Steenwijk, 1999; Jeng, 2004) face extreme difficulties. Further, networks of resistors may serve as a didactic example for introducing the Green's function method, as well as other basic concepts (such as the reciprocal lattice and the Brillouin zone) used in solid state physics. This problem can also be relevant to many other problems, such as random walks (Doyle \& Snell, 1984). 
The perturbation of uniformly tiled resistor lattices by replacing one resistor by another one in the perfect lattice (Owaidat et al., 2014) or removing one resistor from the perfect lattice (Cserti et al., 2002) can be applied to the above Archimedean lattices of resistors.

Finally, the present work can be extended to study the classical lattice dynamics and the vibrational modes of atoms in the harmonic approximation as another application of the Green's function approach.

\section{References}

Asad, J. H., Diab, A. A., Owaidat, M. Q., Hijjawi, R. S., \& Khalifeh, J. M. (2014). Infinite Body Centered Cubic Network of Identical Resistors. Acta Physica Polonica A. http://dx.doi.org/10.12693/APhysPolA.125.60.

Atkinson, D., \& van Steenwijk. F. J. (1999). Infinite resistive lattices. Am. J. Phys., 67, 486-492. http://dx.doi.org/10.1119/1.19311

Cserti, J. (2000). Application of the lattice Green's function for calculating the resistance of an infinite network of resistors. Am. J. Phys., 68, 896-906. http://dx.doi.org/10.1119/1.1419104

Cserti, J., David. G., \& Piroth, A. (2002). Perturbation of infinite networks ofresistors. Am. J. Phys., 70, 153-159. http://dx.doi.org/10.1119/1.1419104

Cserti, J., Szechenyi, G., \& David, G. (2011). Uniform tiling with electrical resistors. J. Phys. A: Math. Theor., 44, 215201-215220. http://dx.doi.org/10.1088/1751-8113/44/21/215201

Doyle, P. G., \& Snell. J. L. (1984). Random Walks and Electric Networks (USA: The Mathematical Association of America).

Jeng, M. (2004). Random walks and effective resistances on toroidal and cylindrical grids. Am. J. Phys., 68(1) 37-40. http://dx.doi.org/10.1119/1.19370

Kirchhoff, G. R. (1847). ÜberdieAuflo“sung der Gleichungen, auf welche man bei der Untersuchung Derlinearen Verteilunggal vanischerStr"omegef" uhrtwird. Ann. Phys. Chem., 72, 497-508.

Owaidat, M. Q, Hijjawi, R. S, \& Khalifeh. J. M. (2012). Network with Two Extra Interstitial Resistors. Int. J. Theor. Phys., 51, 3152. http://dx.doi.org/10.1007/s10773-012-1196-5.

Owaidat, M. Q, Hijjawi. R. S, Asad, J. H, \& Khalifeh. J. M. (2013). Electrical networks with interstitial single capacitor. Mod. Phys. Lett. B, 27, 1350123. http://dx.doi.org/10.1142/S0217984913501236

Owaidat, M. Q, Hijjawi. R. S., \& Khalifeh, J. M. (2014). Perturbation theory of uniform tiling of space with resistors. Eur. Phys. J. Plus., 129, 29. http://dx.doi.org/10.1140/epjp/i2014-14029-y.

Owaidat, M. Q. (2012). Networks of Identical Capacitors with a Substitutional Capacitor. JJP, 5(3), 113-118.

Owaidat, M. Q. (2013). Resistance calculation of the face-centered cubic lattice: Theory and Experiment. Am. J. Phys., 81, 918. http://dx.doi.org/10.1119/1.4826256.

Owaidat, M. Q., Hijjawi, R. S., \& Khalifeh. J. M. (2010). Substitutional single resistor in an infinite square lattice application to lattice Green's function. Mod. Phys. Lett. B, 24, 2057-2068. http://dx.doi.org/10.1142/S021 7984910024468

Owaidat. M. Q., Hijjawi. R. S., \& Khalifeh. J. M. (2010).Interstitial single resistor in a network application of lattice Green's function. J. Phys. A: Math. Theor., 43, 375204-375215. http://dx.doi.org/10.1088/1751-8113/ 43/37/375204.

Tzeng, W. J, \& Wu, F. Y. (2006). Theory of impedance networks: the two-point impedance and $L C$ resonances. $J$. Phys. A: Math. Gen., 39, 8579. http://dx.doi.org/10.1088/0305-4470/39/27/002.

Venezian, G. (1994). On the resistance between two points on a grid. Am. J. Phys., 62, 1000-1004. http://dx.doi.org/10.1119/1.17696

Wu, F. Y. (2004). Theory of resistor networks: the two-point resistance. J. Phys. A: Math. Gen., 37, 6653-6673. http://dx.doi.org/10.1088/0305-4470/37/26/004.

\section{Copyrights}

Copyright for this article is retained by the author(s), with first publication rights granted to the journal.

This is an open-access article distributed under the terms and conditions of the Creative Commons Attribution license (http://creativecommons.org/licenses/by/3.0/). 